\title{
Pelvic reconstruction with different rod-screw systems following Enneking type I/I + IV resection: a clinical study
}

\author{
Peng Lin ${ }^{1,2, *}$, Youyou Shao ${ }^{3, *}$, Huigen Lu ${ }^{1,4}$, Zhengliang Zhang ${ }^{1,5}$, Haiqing Lin ${ }^{1,4}$, \\ Shengdong Wang ${ }^{1}$, Binghao Li ${ }^{1}$, Hengyuan Li ${ }^{1}$, Zhan Wang ${ }^{1}$, Nong Lin ${ }^{1}$, Zhaoming Ye ${ }^{1}$ \\ ${ }^{1}$ Department of Orthopedics, The Second Affiliated Hospital of Zhejiang University School of Medicine/Orthopedics Research \\ Institute of Zhejiang University, Hangzhou 310009, China \\ ${ }^{2}$ Department of Orthopedics, Taizhou Hospital of Zhejiang Province, Linhai 317000, China \\ ${ }^{3}$ Department of Pediatric Pulmonology, The Second Affiliated Hospital and Yuying Children's Hospital of Wenzhou Medical \\ University, Wenzhou 325027, China \\ ${ }^{4}$ Department of Orthopedics, The Second Hospital of Jiaxing, Jiaxing 314000, China \\ ${ }^{5}$ Department of Orthopedics, Dongyang People's Hospital, Jinhua 322100, China \\ *These authors contributed equally to this work
}

Correspondence to: Zhaoming Ye, email: yezhaomingz2@163.com

Keywords: Enneking type I/I + IV, limb salvage surgery, mechanical failure, pelvic girdle reconstruction, pelvic tumor Received: March 06, 2017

Accepted: April 03, 2017

Published: April 17, 2017

Copyright: Peng Lin et al. This is an open-access article distributed under the terms of the Creative Commons Attribution License 3.0 (CC BY 3.0), which permits unrestricted use, distribution, and reproduction in any medium, provided the original author and source are credited.

\section{ABSTRACT}

The mechanical outcomes of patients with pelvic bone tumors involving zone I or zone I + IV who received resection and different reconstructions are not clear. Therefore, the purpose of this study was to compare the outcomes of different rodscrew systems in reconstruction for these patients, and evaluate the relative risk of mechanical failure for them. We reviewed 30 patients for a mean duration of 40.4 months of follow-up (range, 13.1-162.2 months), five patients had mechanical complications. The mechanical survival rate of two-rod and four-screw (TRFS) group was significantly higher than one-rod and two-screw (ORTS) group $(p=0.000)$. The implant survival rate was correlated with ages $(p=0.010)$, younger people are more likely to fail. Thus, TRFS fixation for pelvic reconstruction after Enneking type I/I + IV resection can provide better short to long-term mechanical stability compared with ORTS fixation, the strength of ORTS fixation is not enough. In addition, biological reconstruction such as autologous bone graft is recommended for the patients who are younger or suffered from benign tumor. As for the patients who are older, with malignant tumors, underwent adjuvant radiotherapy or chemotherapy, functional reconstruction with bone cement is a good choice.

\section{INTRODUCTION}

Reconstruction after resection of pelvic bone tumors involving zone I or zone I + IV remains one of the most demanding procedures. A number of authors recommend limb salvage which can provides better quality of life compared with hemipelvectomy, in spite of the risk of local recurrence [1-3]. Limb-salvage surgeries for pelvic tumors were challenging procedures [4], but a great deal of clinical experience, along with the developments of imaging, adjuvant therapies, surgical techniques and reconstruction materials, have proved such kind of surgeries is feasible for selected patients [5-8]. The methods of the reconstruction of the pelvic girdle are not unified, including simple excision without reconstruction $[4,9]$, different rod and screw systems [10-13], bone grafts [14-16], bone cement with the plates or cortical bone screws [17, 18], prosthesis [19], etc. The challenge in reconstruction is to providing a solid reconstitute of pelvic girdle and reduce collapse or rotation of the residual portion of the hemipelvis after weight-bearing and remain good function not only in short term but also in long term follow up. Among multifarious reconstruction methods after internal hemipelvectomy (type I or type I + IV), stable internal fixation and pelvic girdle reconstruction allowed early ambulation and provide better short-term 
to long-term outcomes and function, although the postoperative complications are common [4-7, 20].

Nevertheless, no previous clinical studies have focused on the difference between reconstruction of the pelvic girdle with different rod-screw systems. The purpose of this retrospective study was to review our experience with thirty consecutive cases that underwent reconstructions after Enneking type I/I + IV resection. And to describe the reconstruction with different rodscrew systems, report on the complications and outcomes, evaluate the relative risk of mechanical failure.

\section{RESULTS}

\section{Patients' demographics, post-operative complications}

The mean duration of follow-up was 40.4 months (range, 13.1-162.2 months) at the time of the latest follow-up. The complication rate was about $40 \%$ with 12 patients affected. Three had wound complications, one had superficial infection, none of which required further surgery. Four had neurological defects because of the resection of some sacral nerve roots during type IV resection, only one patient (case 4) had almost compensated two years post-operatively. One was found bone nonunion 65.8 months post-operative, with no mechanical failure.

\section{Oncological results}

Eight patients with benign tumors had no local recurrence and distant metastasis. When considering only malignant tumors, the overall metastasis rate was $40.9 \%$ $(9 / 22)$, the local recurrence rate was $45.5 \%(10 / 22)$.

\section{Mechanical outcomes}

Instrumental survival status were as follows: five patients had mechanical failures and only two patients (case 4,25 ) had a revision surgery. Case 4 had a S1 screw breakage (Figure 1B), and the implant was stabbed to the skin when she stooped, in order to improve the stability, the construction was revised and augmented with an autologous fibular graft (Figure 1C). Case 25 had malposition of a loosening rod (Figure 2B), he had a revision surgery to remove the implant (Figure 2C). Case 26 had implant loosening 3 months post-operatively (Figure 3B), and had implant breakage 51.3 months postoperatively (Figure 3C). There was a pseudoarthrosis formation in the resection site and degeneration development in the symphysis pubis at last follow up, she complained of pain in the resection site and symphysis pubis when walking. Case 22 found implant loosening 20.7 months post-operatively, he walked with crutches from then on, and had no further implant breakage. Case
28 found implant loosening 14 months post-operatively (Figure 4B), he stayed in bed for one month and walked with crutches for 3 months, and had no further implant loosening or breakage. The last three patients have varying degrees of dysfunction, but still can be tolerated, so no further surgery was performed.

Fourteen patients were alive with no mechanical failure, 11 were died without mechanical failure. The cumulative probability of mechanical failure was $11.1 \%$ (95\% confidence interval, $1.6 \%$ to $56.7 \%$ ), $11.1 \%$ (95\% confidence interval, $1.6 \%$ to $56.7 \%$ ), $25.9 \%$ (95\% confidence interval, $7 \%$ to $71.1 \%$ ), 50.6\% (95\% confidence interval, $17.7 \%$ to $92.2 \%)$, and $75.3 \%(95 \%$ confidence interval, $34.8 \%$ to $99.0 \%$ ) at six, twelve, eighteen, twenty-four and thirty-six months, respectively in ORTS group. The cumulative probability of mechanical failure was $0 \%$ (95\% confidence interval, $0 \%$ to $0 \%$ ) at one to five years, and 20\% (95\% confidence interval, $3.1 \%$ to $79.6 \%$ ) at six to thirteen years, respectively in TRFS group. The implant survival rate was statistically influenced by the reconstruction with different rod-screw systems. The survival rate of TRFS group was higher than ORTS group ( $p=0.000)$, the cumulative implant survival cures of the two groups is shown in Figure 5.

We then compare the overall implant survival rate between the five sub groups (described above), there was statistically difference between each group by the pooled over strata test $(p=0.008)$. We compared the difference between each two groups by pairwise for each stratum test, and found statistically difference between extrapelvic and intrapelvic + extrapelvic group $(p=0.001)$, intrapelvic and intrapelvic + extrapelvic group $(p=0.020)$, extrapelvic and intrapelvic + intrapelvic group $(p=0.034)$. There was no statistically difference between extrapelvic and intrapelvic group $(p=0.910)$.

The implant survival rate of zone I + IV group was lower than zone I group $(p=0.022)$, there was no statistical difference if exclude the influence of different rod-screw group factor $(p=0.447)$. There was no overall statistically difference between the use of bonegraft, bone cement and none $(p=0.054)$, but the survival rate with bone graft is higher than none $(p=0.033)$. The implant survival rate was correlated with ages by linear trend test $(p=0.010)$, younger people are more likely to fail. The implant survival rate in group of ages less than 22 was lower than group of ages larger than $22(p=0.000)$. It was not statistically influenced by chemotherapy ( $p=0.085)$, radiotherapy $(p=0.150)$, gender $(p=0.730)$ or BMI $(p=0.317)$.

\section{Functional outcomes}

The mean Musculoskeletal Tumor Society [21] (MSTS) score (\%) post-operatively was $81.0 \%$ (range, 43 to $97 \%$ ). The mean MSTS score (\%) of the mechanical failure patients post-operatively was $59.4 \%$ (SD: $13.5 \%$ ), the mean MSTS score (\%) of the patients with no 
mechanical failure post-operatively was $85.3 \%$ (SD: $9.6 \%$ ). The MSTS score of the patients with no mechanical failure was significantly higher than mechanical failure patients $(p=0.000)$. The mean MSTS score $(\%)$ of the ORTS patients post-operatively was $73.1 \%$ (SD: $18.5 \%$ ), the mean MSTS score ( $\%$ ) of the TRFS patients post-operatively was $84.4 \%$ (SD: $10.5 \%$ ), there was no significantly difference between these two groups $(p=0.116)$.

\section{Risk factors of post-operative mechanical failures}

To investigate the risk factors of post-operative mechanical failures, we firstly did a univariate correlation analysis including 10 factors listed in Table 1 and Table 2. We found that reconstruction with ORTS system was a risk factor (Table 3), as the relative risk was $16(95 \%$
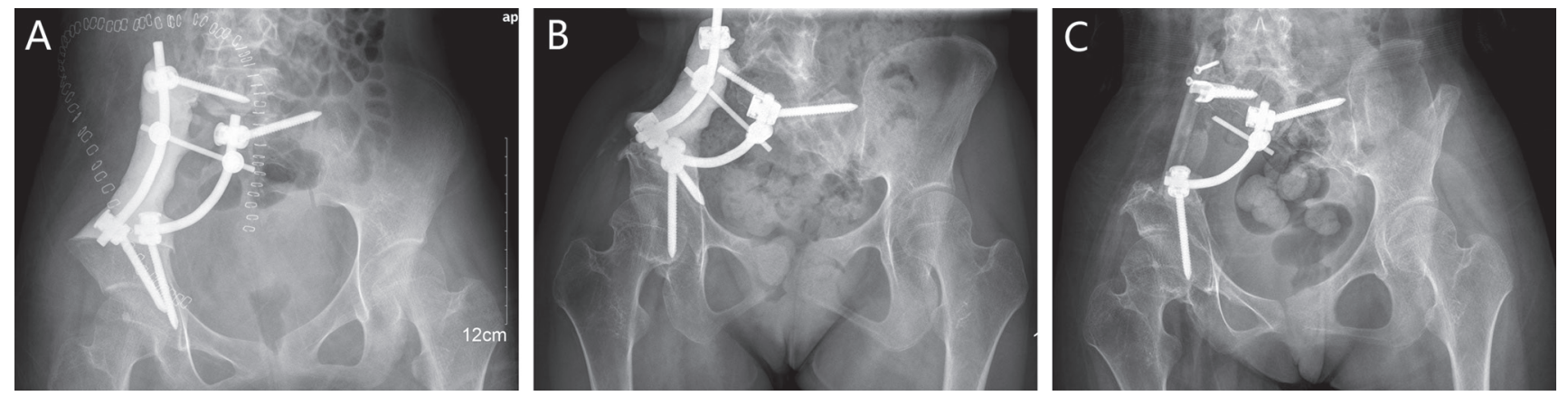

Figure 1: A 16-year-old female (case 4) with diagnosis of pelvis osteosarcoma affecting zone I + IV. (A) AP radiograph at one week post-operatively. (B) AP radiograph at 61.9 months post-operatively, showing failure of the implant, and the implant was stabbed to the skin when she stooped, (C) AP radiograph after the construction was revised and augmented with an autologous fibular graft 80 months post-operatively.
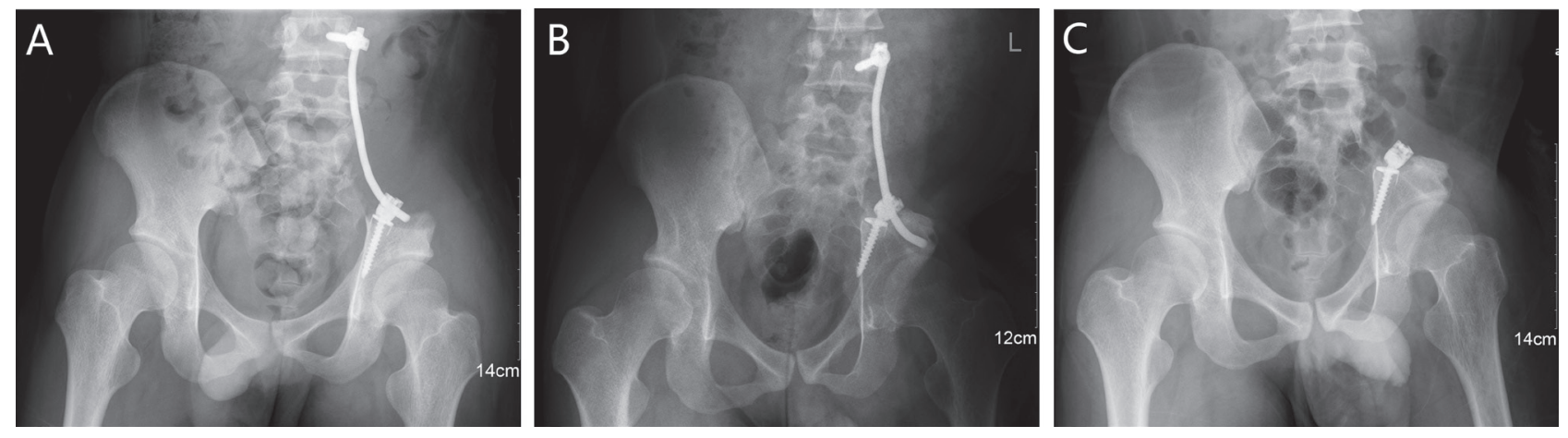

Figure 2: A 20-year-old male (case 25) with diagnosis of pelvis osteosarcoma affecting zone I. (A) AP radiograph at two weeks post-operatively. (B) AP radiograph at 30.5 months post-operatively, showing failure of the implant and with functional impairment. (C) AP radiograph at one week after removal of the implant.
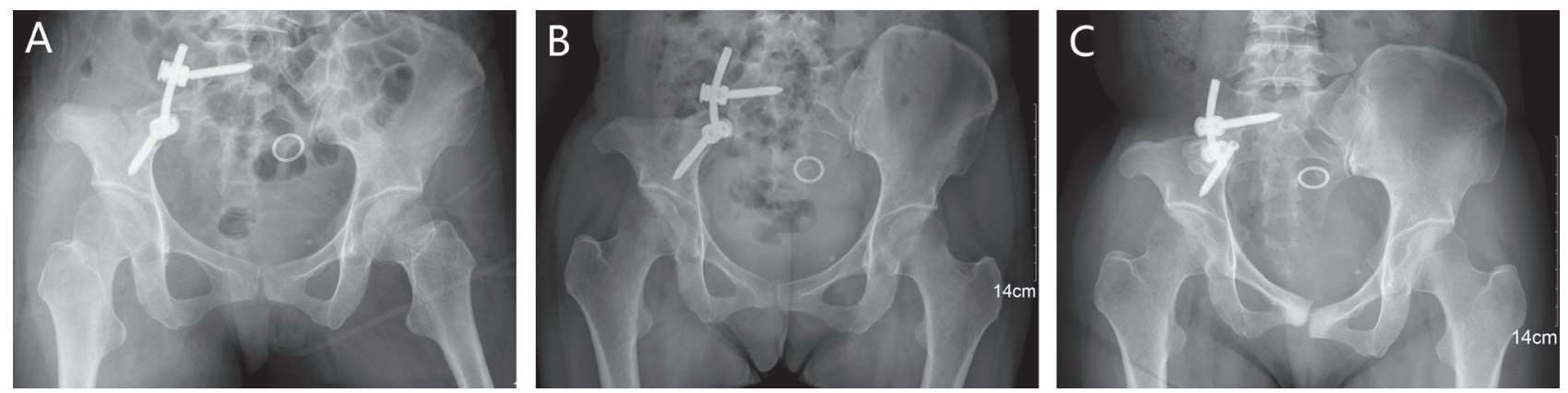

Figure 3: A 37-year-old female (case 26) with diagnosis of pelvis chondrosarcoma affecting zone I. (A) AP radiograph of the pelvis after bone tumor resection and reconstruction with an autograft bone and screw-rod system. (B) AP radiograph showing the implant loosening 3 months post-operatively without functional impairment. (C) at 51.3 months post-operatively, AP radiograph showing failure of the implant, pseudoarthrosis formation was found in the resection site, and degeneration was developed in the symphysis pubis. 
confidence interval, 1.45 to 176.45). Age less than 22 was a risk factor (Table 3), as the relative risk was 96 (95\% confidence interval, 4.94 to 1865.70$)$. Zone IV involvement may a potential risk factor $(p=0.022)$ though there was no statistical difference if exclude the influence of different rod-screw group factor $(p=0.447)$, the relative risk was 11 (95\% confidence interval, 1.27 to 95.18$)$.

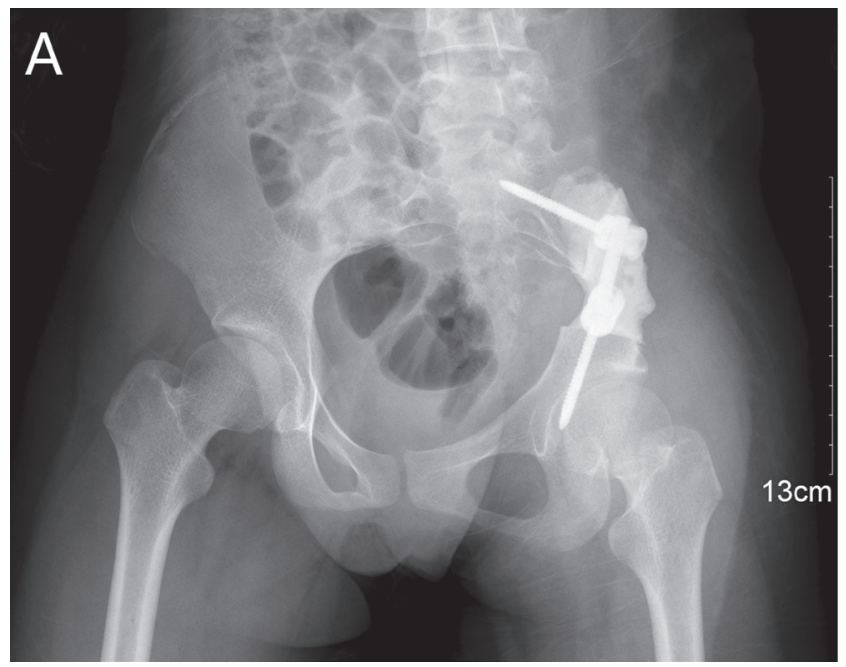

\section{DISCUSSION}

The traditional hemipelvectomy in treating pelvic tumors was replaced by limb-salvage surgery, which was combined with adjuvant therapy since the similar survival and recurrence rates [22-24]. Limb-salvage surgery can improve quality of life, reduce psychological trauma and physical disability, which becomes a favorable procedure.

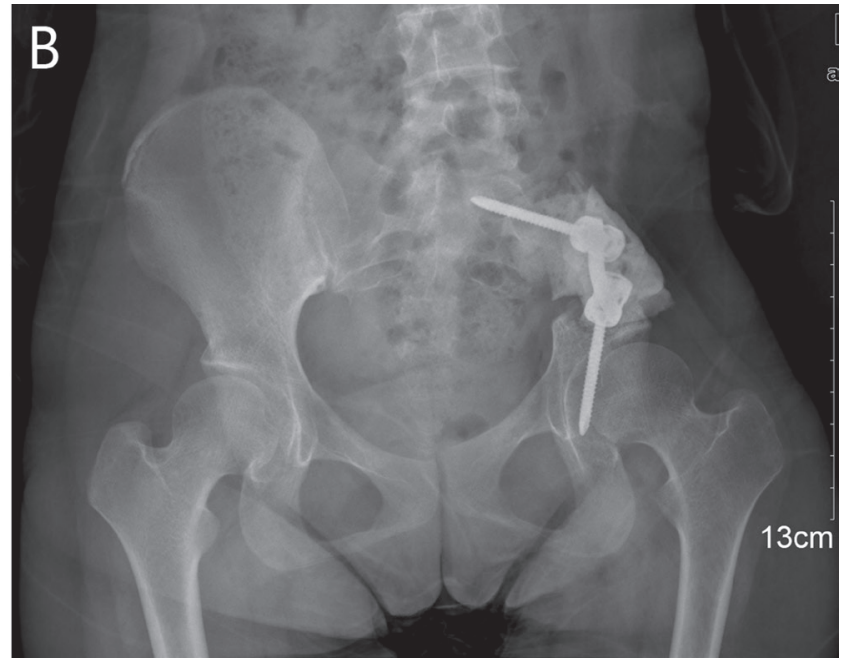

Figure 4: A 15-year-old female (case 28) with diagnosis of pelvis osteosarcoma affecting zone I. (A) AP radiograph of the pelvis after bone tumor resection and reconstruction with screw-rod system and bone cement. (B) AP radiograph showing the implant loosening 14 months post-operatively without functional impairment.

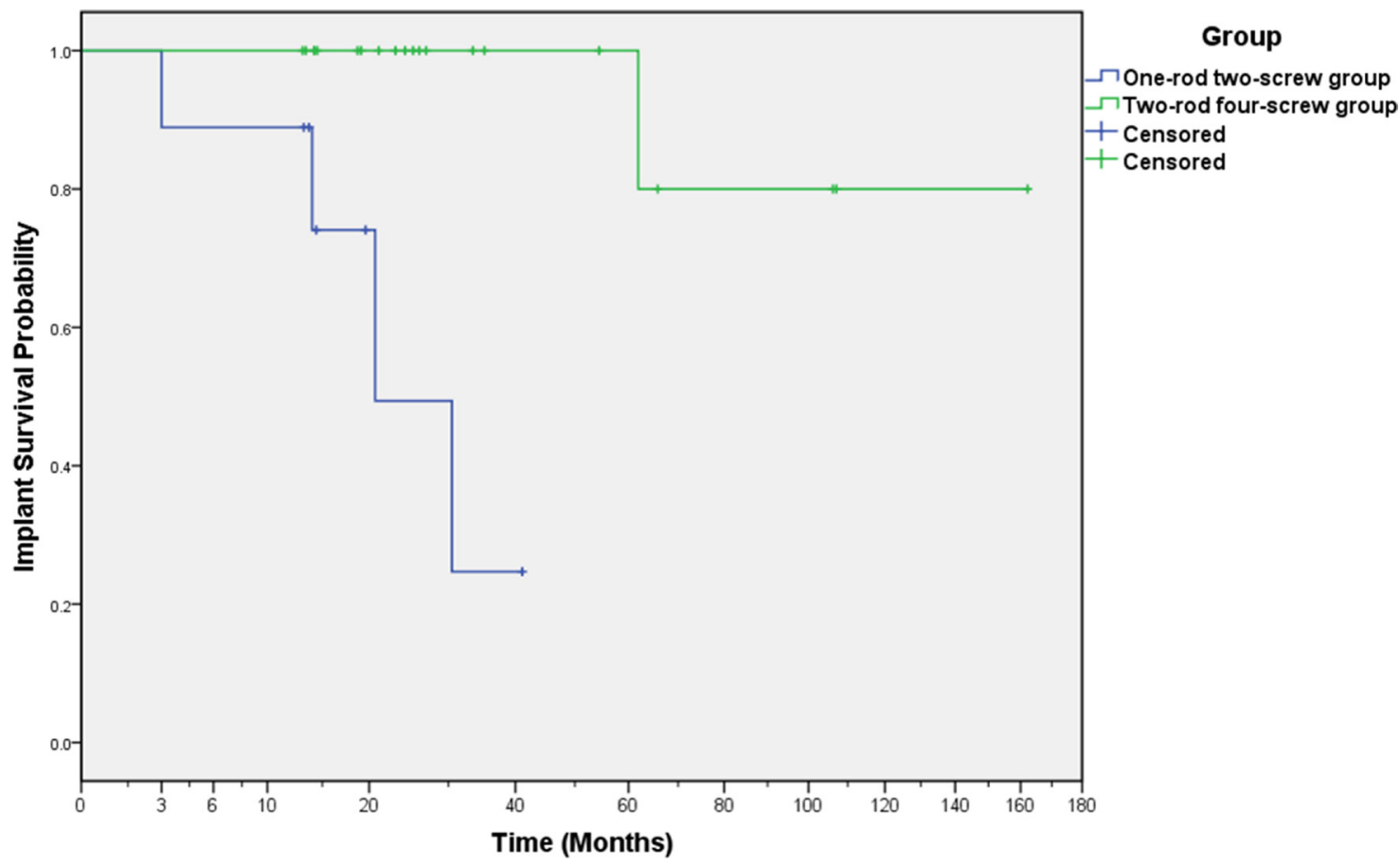

Figure 5: Overall implant survival analysis through Kaplan-Meier for different reconstruction method. Number of patients: 30 patients. A statistically significant difference was noted between the one-rod two-screw group and the two-rod four-screw group $(p=0.000)$. 
Table 1: Patient demographics data and adjuvant therapy details

\begin{tabular}{|c|c|c|c|c|c|c|c|c|c|c|}
\hline No. & Age & Gender & $\begin{array}{c}\text { Height } \\
\text { (cm) }\end{array}$ & $\begin{array}{c}\text { Weight } \\
\text { (kg) }\end{array}$ & BMI & Histologic diagnosis & Bone graft & $\begin{array}{c}\text { Bone } \\
\text { cement }\end{array}$ & Chemotherapy & Radiotherapy \\
\hline 1 & 26 & Female & 158 & 40.5 & 16.2 & Osteoclastoma & None & Yes & None & None \\
\hline 2 & 35 & Male & 174 & 70 & 23.1 & Osteoclastoma & Yes & None & None & None \\
\hline 3 & 48 & Female & 158 & 53 & 21.2 & Non-ossifying fibroma & Yes & None & None & None \\
\hline 4 & 16 & Female & 150 & 34 & 15.1 & Osteosarcoma & None & Yes & Yes & None \\
\hline 5 & 41 & Male & 175 & 65 & 21.2 & Chondrosarcoma & Yes & None & None & None \\
\hline 6 & 44 & Female & 162 & 49 & 18.7 & Osteoclastoma & Yes & None & None & None \\
\hline 7 & 39 & Male & 168 & 60 & 21.3 & Chondrosarcoma & Yes & None & None & None \\
\hline 8 & 63 & Female & 149 & 52 & 23.4 & Chondrosarcoma & Yes & None & None & None \\
\hline 9 & 33 & Male & 173 & 65 & 21.7 & Chondrosarcoma & None & Yes & None & None \\
\hline 10 & 59 & Male & 174 & 53 & 17.5 & Osteosarcoma & None & Yes & Yes & None \\
\hline 11 & 38 & Male & 165 & 55 & 20.2 & Benign & Yes & None & None & None \\
\hline 12 & 68 & Male & 177 & 63 & 20.1 & Chondrosarcoma & None & Yes & None & None \\
\hline 13 & 43 & Female & 161 & 61 & 23.5 & Osteosarcoma & Yes & None & Yes & None \\
\hline 14 & 48 & Female & 164 & 47 & 17.5 & Metastatic Thyroid cancer & None & Yes & None & Yes \\
\hline 15 & 66 & Male & 172 & 58 & 19.6 & Metastatic bladder cancer & None & Yes & None & None \\
\hline 16 & 36 & Female & 164 & 55 & 20.4 & Osteoclastoma & None & Yes & None & None \\
\hline 17 & 69 & Female & 167 & 55 & 19.7 & Metastatic cancer & None & Yes & Yes & None \\
\hline 18 & 15 & Male & 165 & 35 & 12.9 & Chondrosarcoma & Yes & None & Yes & None \\
\hline 19 & 59 & Female & 164 & 48 & 17.8 & Osteosarcoma & None & None & Yes & None \\
\hline 20 & 28 & Male & 174 & 52 & 17.2 & Chondrosarcoma & Yes & None & None & None \\
\hline 21 & 44 & Female & 165 & 55 & 20.2 & Malignant fibrous histiocytoma & None & None & Yes & None \\
\hline 22 & 19 & Male & 172 & 60 & 20.3 & Fibrosarcoma & None & None & None & None \\
\hline 23 & 34 & Male & 172 & 55 & 18.6 & Solitary fibrous tumor & Yes & None & None & None \\
\hline 24 & 24 & Male & 173 & 56 & 18.7 & Ewing's sarcoma & Yes & None & Yes & Yes \\
\hline 25 & 20 & Male & 172 & 64 & 21.6 & Osteosarcoma & None & None & Yes & None \\
\hline 26 & 37 & Female & 165 & 60 & 22.0 & Chondrosarcoma & Yes & None & None & None \\
\hline 27 & 32 & Female & 161 & 50 & 19.3 & Chondrosarcoma & Yes & None & None & None \\
\hline 28 & 15 & Female & 159 & 50 & 19.8 & Osteosarcoma & None & Yes & Yes & Yes \\
\hline 29 & 61 & Male & 165 & 55 & 20.2 & Aneurysmal bone cyst & None & Yes & None & None \\
\hline 30 & 62 & Male & 174 & 58 & 19.2 & Metastatic malignant melanoma & None & None & Yes & None \\
\hline
\end{tabular}

Table 2: Surgical details, complications and the outcomes of the patients

\begin{tabular}{|c|c|c|c|c|c|c|c|c|c|}
\hline No. & $\begin{array}{l}\text { Type of } \\
\text { resection }\end{array}$ & $\begin{array}{l}\text { Site of fixation (proximal/ } \\
\text { distal) }\end{array}$ & Method of fixation & $\begin{array}{l}\text { Type of } \\
\text { fixation }\end{array}$ & $\begin{array}{l}\text { MSTS } \\
\text { score } \\
(\%)\end{array}$ & Complications & $\begin{array}{l}\text { mechanical } \\
\text { survival } \\
\text { time } \\
\text { (months) }\end{array}$ & $\begin{array}{l}\text { Mechanical } \\
\text { outcome }\end{array}$ & $\begin{array}{l}\text { Follow-up } \\
\text { period (months) }\end{array}$ \\
\hline 1 & I & $\begin{array}{l}\text { Vertebral pedicle (L5), } \\
\text { sacrum/ periacetabular bone }\end{array}$ & 2 rods 4 screws & $\mathrm{EXP}+\mathrm{INP}$ & 97 & & 162.2 & $\mathrm{AMF}$ & 162.2 \\
\hline 2 & I & $\begin{array}{l}\text { Transverse process (L5), } \\
\text { sacrum/ periacetabular bone }\end{array}$ & 2 rods 4 screws & $\mathrm{EXP}+\mathrm{INP}$ & 90 & & 107.1 & AMF & 107.1 \\
\hline 3 & I & $\begin{array}{l}\text { Transverse process (L5, } \\
\text { sacrum)/periacetabular bone }\end{array}$ & 2 rods 4 screws & $\mathrm{EXP}+\mathrm{INP}$ & 90 & & 106.2 & $\mathrm{AMF}$ & 106.2 \\
\hline 4 & $\mathrm{I}+\mathrm{IV}$ & $\begin{array}{l}\text { Transverse process (L5), } \\
\text { sacrum/ periacetabular bone }\end{array}$ & 2 rods 4 screws & $\mathrm{EXP}+\mathrm{INP}$ & 77 & $\begin{array}{l}\text { Neurological defects, } \\
\text { metal breakage }\end{array}$ & 61.9 & MF & 84.5 \\
\hline 5 & I & $\begin{array}{l}\text { Transverse process (L5), } \\
\text { sacrum/periacetabular bone }\end{array}$ & 2 rods 4 screws & $\mathrm{EXP}+\mathrm{INP}$ & 87 & Bone nonunion & 65.8 & DMF & 65.8 \\
\hline
\end{tabular}




\begin{tabular}{|c|c|c|c|c|c|c|c|c|c|}
\hline 6 & I & $\begin{array}{l}\text { Transverse process sacrum/ } \\
\text { periacetabular bone }\end{array}$ & 2 rods 4 screws & INP + INP & 97 & & 54.4 & AMF & 54.4 \\
\hline 7 & I & $\begin{array}{l}\text { Transverse process sacrum/ } \\
\text { periacetabular bone }\end{array}$ & 2 rods 4 screws & INP + INP & 87 & Superficial infection & 35.2 & DMF & 35.2 \\
\hline 8 & I & $\begin{array}{l}\text { Vertebral pedicle (L5), } \\
\text { sacrum/ periacetabular bone }\end{array}$ & 2 rods 4 screws & INP+EXP & 87 & & 33.5 & AMF & 33.5 \\
\hline 9 & I & $\begin{array}{l}\text { Transverse process (L5), } \\
\text { sacrum/periacetabular bone }\end{array}$ & 2 rods 4 screws & INP+EXP & 83 & & 27.0 & DMF & 27.0 \\
\hline 10 & I & $\begin{array}{l}\text { Transverse process (L5), } \\
\text { sacrum/ periacetabular bone }\end{array}$ & 2 rods 4 screws & EXP + INP & 83 & Wound complication & 26.1 & DMF & 26.1 \\
\hline 11 & I & $\begin{array}{l}\text { Transverse process sacrum/ } \\
\text { periacetabular bone }\end{array}$ & 2 rods 4 screws & $\mathrm{INP}+\mathrm{INP}$ & 93.3 & & 25.3 & $\mathrm{AMF}$ & 25.3 \\
\hline 12 & I & $\begin{array}{l}\text { Transverse process sacrum/ } \\
\text { periacetabular bone }\end{array}$ & 2 rods 4 screws & INP + INP & 87 & & 24.3 & DMF & 24.3 \\
\hline 13 & I & $\begin{array}{l}\text { Transverse process (L5), } \\
\text { sacrum/ periacetabular bone }\end{array}$ & 2 rods 4 screws & EXP + INP & 90 & & 23.1 & DMF & 23.1 \\
\hline 14 & I & $\begin{array}{l}\text { Transverse process sacrum/ } \\
\text { periacetabular bone }\end{array}$ & 2 rods 4 screws & INP + INP & 63.3 & & 21.1 & DMF & 21.1 \\
\hline 15 & I & $\begin{array}{l}\text { Transverse process sacrum/ } \\
\text { periacetabular bone }\end{array}$ & 2 rods 4 screws & $\mathrm{INP}+\mathrm{INP}$ & 80 & Wound complication & 14.5 & $\mathrm{AMF}$ & 14.5 \\
\hline 16 & I & $\begin{array}{l}\text { Transverse process } \\
\text { (L5),sacrum/periacetabular } \\
\text { bone }\end{array}$ & 2 rods 4 screws & EXP + INP & 90 & & 14.2 & AMF & 14.2 \\
\hline 17 & I & $\begin{array}{l}\text { Transverse process sacrum/ } \\
\text { periacetabular bone }\end{array}$ & 2 rods 4 screws & $\mathrm{INP}+\mathrm{INP}$ & 77 & & 14.2 & DMF & 14.2 \\
\hline 18 & I & $\begin{array}{l}\text { Vertebral pedicle (L5), } \\
\text { sacrum/ periacetabular bone }\end{array}$ & 2 rods 4 screws & EXP + INP & 97 & & 13.1 & AMF & 13.1 \\
\hline 19 & $\mathrm{I}+\mathrm{IV}$ & $\begin{array}{l}\text { Vertebral pedicle (L5, S1)/ } \\
\text { periacetabular bone }\end{array}$ & 1 rod 4 screws & EXP + EXP & 67 & $\begin{array}{l}\text { Neurological } \\
\text { defects, deep venous } \\
\text { thrombosis }\end{array}$ & 19.1 & AMF & 19.1 \\
\hline 20 & I & $\begin{array}{l}\text { Vertebral pedicle }(\mathrm{L} 5, \mathrm{~S} 1) / \\
\text { periacetabular bone }\end{array}$ & 1 rod 4 screws & EXP + EXP & 90 & & 18.7 & DMF & 18.7 \\
\hline 21 & $\mathrm{I}+\mathrm{IV}$ & $\begin{array}{l}\text { Vertebral pedicle }(\mathrm{L} 4,5) / \\
\text { periacetabular bone }\end{array}$ & 1 rod 4 screws & EXP + EXP & 60 & Neurological defects & 13.4 & DMF & 13.4 \\
\hline 22 & $I+I V$ & $\begin{array}{l}\text { Vertebral pedicle }(\mathrm{L} 4,5) / \\
\text { periacetabular bone }\end{array}$ & 1 rod 3 screws & EXP & 60 & $\begin{array}{l}\text { Neurological defects, } \\
\text { implant loosening }\end{array}$ & 20.7 & MF & 41.0 \\
\hline 23 & $\mathrm{I}+\mathrm{IV}$ & $\begin{array}{l}\text { Transverse process (L5)/ } \\
\text { periacetabular bone }\end{array}$ & 1 rod 3 screws & EXP & 83 & & 19.6 & AMF & 19.6 \\
\hline 24 & I & $\begin{array}{l}\text { Vertebral pedicle (L5, S1)/ } \\
\text { periacetabular bone }\end{array}$ & 1 rod 3 screws & INP & 87 & & 14.4 & AMF & 14.4 \\
\hline 25 & $\mathrm{I}+\mathrm{IV}$ & $\begin{array}{l}\text { vertebral pedicle (L4)/ } \\
\text { periacetabular bone }\end{array}$ & 1 rod 2 screws & EXP & 43 & Implant loosening & 30.5 & MF & 67.3 \\
\hline 26 & I & $\begin{array}{l}\text { Transverse process sacrum/ } \\
\text { periacetabular bone }\end{array}$ & 1 rod 2 screws & INP & 50 & Metal breakage & 3.0 & MF & 67.2 \\
\hline 27 & I & $\begin{array}{l}\text { Transverse process sacrum/ } \\
\text { periacetabular bone }\end{array}$ & 1 rod 2 screws & INP & 90 & & 41.1 & AMF & 41.1 \\
\hline 28 & I & $\begin{array}{l}\text { Transverse process sacrum/ } \\
\text { periacetabular bone }\end{array}$ & 1 rod 2 screws & INP & 67 & Implant loosening & 14.0 & $\mathrm{MF}$ & 32.1 \\
\hline 29 & I & $\begin{array}{l}\text { Transverse process sacrum/ } \\
\text { periacetabular bone }\end{array}$ & 1 rod 2 screws & INP & 91 & & 13.7 & AMF & 13.7 \\
\hline 30 & I & $\begin{array}{l}\text { Vertebral pedicle (L5)/ } \\
\text { periacetabular bone }\end{array}$ & 1 rod 2 screws & EXP & 87 & Wound complication & 13.2 & DMF & 13.2 \\
\hline
\end{tabular}

INP: intrapelvic, sacral-pelvic reconstruction, EXP: extrapelvic, lumbo-pelvic reconstruction, MSTS: Musculoskeletal Tumor Society, AMF: alive with no mechanical failure, DMF: died with no mechanical failure, MF: mechanical failure.

With the development of surgical technique and implant, although the simple resection without reconstruction has certain effect, it is gradually abandoned. Reconstruction with the screw and rod system is the most common choice on account of its reliability and effectiveness. However, mechanical failure was a common complication following internal hemipelvectomy, no matter which reconstruction method has been chosen $[9,10,12,25]$. To date, there are only two articles using finite element analysis of the biomechanics to evaluate different methods of rod-screw systems [26, 27]. In our cohort, we compared the effects of different rod-screw fixations on the prognosis, and found 
Table 3: Potential risk factors of post-operative implant failures in univariate analysis

\begin{tabular}{|c|c|c|c|}
\hline Variables & Mechanical failure No. (\%) & Non-mechanical failure No. (\%) & $p$ value \\
\hline Rod-screw system & & & $0.000^{*}$ \\
\hline One-rod two-screw (ORTS) & $4(44.4)$ & $5(55.6)$ & $0.008^{* *}$ \\
\hline EXP & $2(50)$ & $2(50)$ & $0.910^{\#}$ \\
\hline INP & $2(40)$ & $3(60)$ & \\
\hline Two-rod four-screw (TRFS) & $1(4.8)$ & $20(95.2)$ & \\
\hline $\mathrm{EXP}+\mathrm{EXP}$ & $0(0)$ & $3(100)$ & \\
\hline $\mathrm{INP}+\mathrm{EXP}$ & $1(9.1)$ & $10(90.9)$ & $0.001^{\# \#}$ \\
\hline INP + INP & $0(0)$ & $7(100)$ & $0.034^{\# \#}$ \\
\hline Tumor location & & & 0.022 \\
\hline I & $2(8.3)$ & $22(91.7)$ & \\
\hline $\mathrm{I}+\mathrm{IV}$ & $3(50)$ & $3(50)$ & \\
\hline Reconstruction & & & 0.054 \\
\hline Bone graft & $1(7.1)$ & 13 (92.9) & $0.033^{\# \# \#}$ \\
\hline Bone cement & $2(18.2)$ & $9(81.8)$ & $0.122^{\# \#}$ \\
\hline None & $2(40)$ & $3(60)$ & \\
\hline Chemotherapy & & & 0.085 \\
\hline Yes & $3(27.3)$ & $8(72.7)$ & \\
\hline None & $2(10.5)$ & $17(89.5)$ & \\
\hline Radiotherapy & & & 0.150 \\
\hline Yes & $1(33.3)$ & $2(66.7)$ & \\
\hline None & $4(14.8)$ & $23(85.2)$ & \\
\hline Gender & & & 0.730 \\
\hline Male & $2(12.5)$ & $14(87.5)$ & \\
\hline Female & $3(21.4)$ & $11(78.6)$ & \\
\hline Age (years) & & & 0.000 \\
\hline$<22$ & $4(80)$ & $1(20)$ & \\
\hline$>22$ & $1(4)$ & $24(96)$ & \\
\hline BMI & & & 0.317 \\
\hline$<19.75$ & $1(7.1)$ & 13 (92.9) & \\
\hline$>19.75$ & $4(25)$ & $12(75)$ & \\
\hline All & $5(16.7)$ & $25(83.3)$ & \\
\hline
\end{tabular}

Significant at a $=0.05$ * significant difference between ORTS and TRFS group, ${ }^{* *}$ significant difference between five sub groups by the pooled over strata test, ${ }^{\#}$ compared with INP group by pairwise for each stratum test, ${ }^{\#}$ compared with EXP group by pairwise for each stratum test, ${ }^{\# \#}$ compared with None group by pairwise for each stratum test. (Kaplan-Meier survival analysis, Log rank test was used to compare implant survival rates between different groups). INP: intrapelvic, sacral-pelvic reconstruction, EXP: extrapelvic, lumbo-pelvic reconstruction.

inadequate fixation of the pelvic girdle is likely the main reason in the mechanical failures.

ORTS fixation has been used by many authors, and achieved good outcomes $[10,11]$. In our series, we adopt the similar method as it reports [10], although there were several satisfactory results (Figure 6B), the total abortion incidence was significantly higher than TRFS fixation, we considered this reconstruction method is insufficient as the 
torsional stability is not enough (Figures 2B, 3B-3C, 4B). One literature introduced TRFS fixation, they believe the mechanical strength of TRFS system is good, of course, if coupled with bone cement or bone graft, the initial stability will be better, this conclusion is similar to our result [13]. We performed the TRFS fixation as previously reported [12], there is only one patients (4.8\%) suffered implant breakage 61.9 months post-operatively when she was 16 years old at the time of the index reconstruction with the bone tumor involving zone I + IV(Figure 1B). We found the implant survival rate was correlated with ages in our cohort $(p=0.010)$, younger people are more likely to fail, because the younger patients with longer survival period, they exercise more, need higher life quality and require better implant intensity. For these younger patients and the primary tumor which has a good life expectancy, biological reconstruction with bone graft can improve their long-term stability.

Intrapelvic fixation is more stable than extrapelvic fixation. The reconstruction of extrapelvic fixation is easy to cause the failure in the long-term follow-up, because of the existence of micromotion between intervertebral discs (i.e., L4/5, L5/S1), the internal fixation stress will increase [27] and the internal fixation will be easy to get fatigue broken or loosening. Case 4 is a good example, there's another case (case 25) in our series, who had the lumbar 4 to iliac bone fixation, the implants loosened 30.5 months post-operatively. We would recommend to achieve intrapelvic fixation if possible mainly for the mechanical stability. But in some cases, it's difficult to put two screws into the sacrum, because the space for the screws in sacrum is limited. Therefore, only one screw could fixed in the sacrum, and the other one is fixed on the lumbar 5 vertebra, as we described as extrapelvic+intrapelvic fixation (Figures 1A, 7A-7C). Zone IV involvement may a potential risk factors $(p=0.022)$, partly because of the extrapelvic fixation due to no enough position for the screws in sacrum. Compared with pedicle screws, lateral lumbar/ sacrum vertebral body screws are recommended, as this front column support has biomechanical advantages [28].

Most of the literatures recommend biological reconstruction, although there is nonunion, bone graft fracture [29], infection [30-32], etc., since its long-term benefits $[10,11,14,15,22,25,33-35]$. When doing the biological reconstruction after resection of pelvic bone tumors involving zone I or zone I $+\mathrm{IV}$, the iliac crest bone graft on the same side of the host is the first choice (gluteus medius muscle pedicle iliac bone graft [10], vascularized [15] or non-vascularized [11] iliac bone graft) (Figure 8A). When compared with the autogenous fibula graft, it has the advantages of convenient to harvest and minimal invasive. Whereas reconstructions with iliac crest have limitations, as the remainder of the iliac bone is limited when requiring extensive bone resection. Bone grafts such as nonvascularized or vascularized fibular grafts are commonly used to reconstruct large bone defects after resection of the tumor (Figure 1C) [14, 25, 33-35]. There is a high rate of infection and nonunion reported in the allograft fibular transplantation $[30,32]$, so we do not recommend this technique as first choice. Excellent outcomes were achieved by reconstruction with vascularized fibular grafts in the literatures [34-36]. Nevertheless, this technique requires more complex procedures, longer operative time, greater surgical trauma, and higher donor site morbidity [37]. Non-vascularized fibular grafts are likely to have lower donor site morbidity and are less complex to implant [25], in spite of reservations a lack of biological activity, risk of resorption [38], and require a slightly longer time to union [39]. As a compromised approach, in our view, non-vascularized fibular grafts are the useful alternative to vascularized grafts, especially in sacral iliac region with adequate blood supply and soft-tissue coverage.

Although biological reconstruction is a mainstream, functional reconstruction with rod-screw system and bone cement is recommended for the patients underwent adjuvant radiotherapy or chemotherapy. Because adjuvant radiotherapy or chemotherapy may cause nonunion after bone graft implant, they may have a negative impact on the stability of the biological reconstruction $[11,14]$.

Several factors need to be considered when selecting the most appropriate reconstructive procedure. For the younger patients, biological reconstruction has great advantages in long term stability (Figure 6B, 8B), compared with non-biological reconstruction (Figure 1B) $[10,14,15$, $25,33-35]$. For the older patients who can't stay in bed for a long time because of poor general condition, bone cement is recommend for the instantaneous mechanical stability, and the patients can get early mobilization [12].

In terms of the oncological outcomes, it was thought that primary or metastatic malignancies, which have a poor life expectancy, should be differentiated from benign tumors. Patient selection criteria, therefore, should take account of oncological outcomes, patients with pelvic malignancies would be recommended to use bone cement mainly for the instantaneous mechanical stability. While treating with benign tumors like osteoclastoma or the primary malignancies which have a good life expectancy like chondrosarcoma, however, autologous bone graft is recommended because this is a biological reconstruction method which has good long-term mechanical stability (Figures 6, 8).

Our study has some limitations such as the limited number of patients, so we include all patients whose data we have collected no matter the length of follow-up. This was however a retrospective studies on patients, so the confounding factors can't be fully controlled. The time of follow up ranged widely, because of the progress of malignant tumor is rapidly. Accordingly, the long-term mechanical failure rate was underestimate the genuine rates.

In conclusion, TRFS fixation for pelvic reconstruction after Enneking type I/I + IV resection 

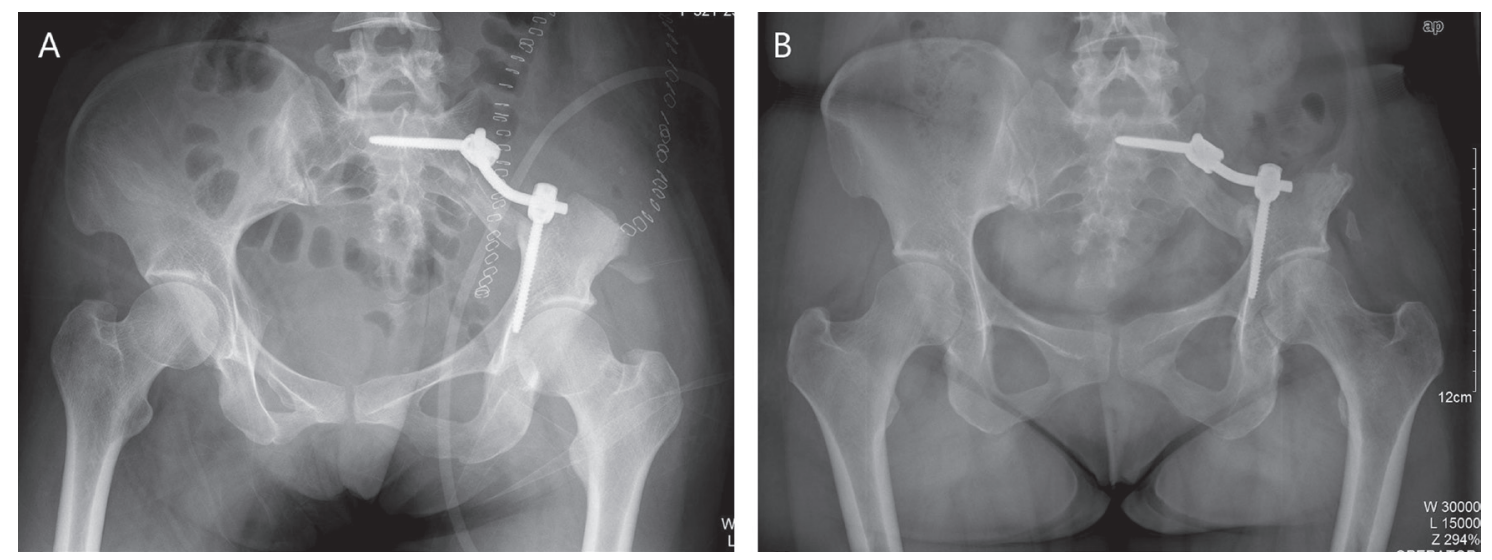

Figure 6: A 32-year-old woman (case 27) with diagnosis of pelvis chondrosarcoma affecting zone I. (A) AP radiograph of the pelvis after internal hemipelvectomy and subsequent pelvic ring reconstruction with screw-rod system and autograft bone. (B) AP radiograph showing the bone union 12.3 months post-operatively.
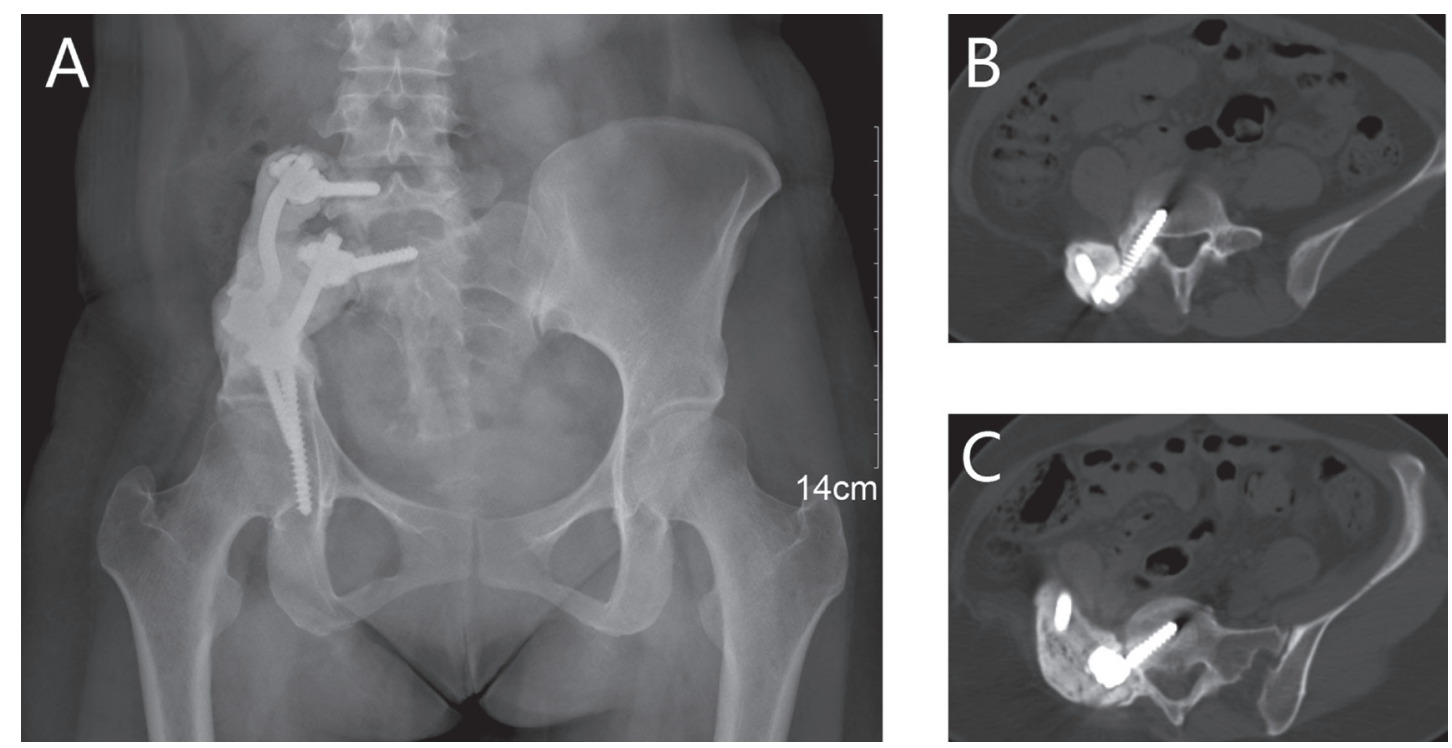

Figure 7: A 26-year-old female (case 1) with diagnosis of pelvis osteoclastoma affecting zone I. (A) AP radiograph of the pelvis shows internal hemipelvectomy and subsequent pelvic ring reconstruction with screw-rod system and bone cement. (B, C) CT images show no evidence of tumor recurrence or implant loosening 144.1 months post-operatively.
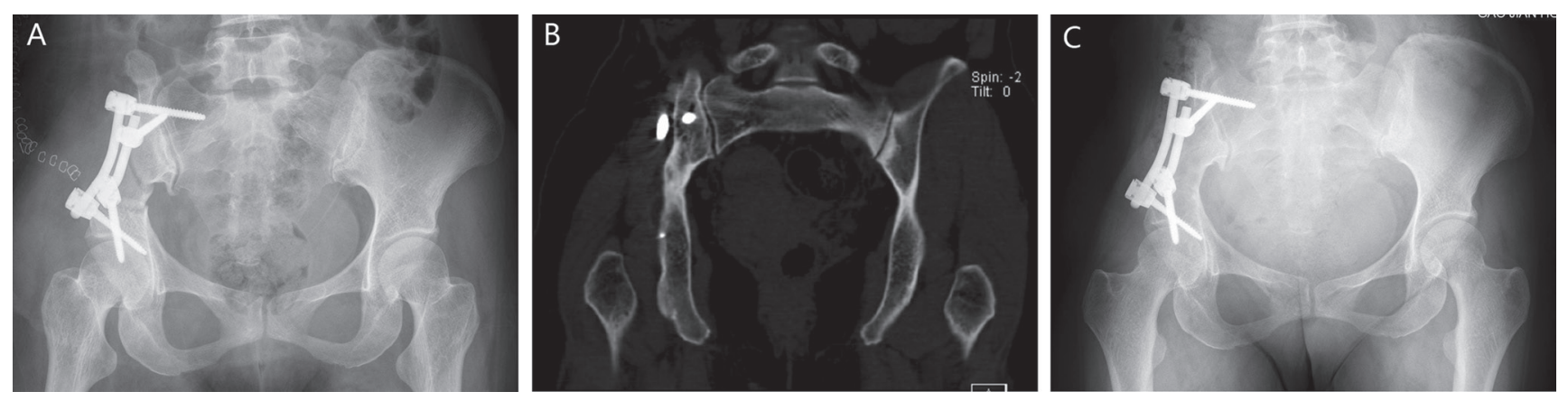

Figure 8: A 44-year-old woman (case 6) with diagnosis of pelvis osteoclastoma affecting zone I. (A) AP radiograph of the pelvis after bone tumor resection and reconstruction with an autograft bone in the pelvis stabilized with the implantation of screw-rod system. (B) Coronal CT image showing the bone union 17.5 months post-operatively. (C) AP radiograph of the pelvis shows no evidence of tumor recurrence or implant loosening 36 months post-operatively. 
can provide better short to long-term mechanical stability compared with ORTS fixation, the strength of ORTS fixation is not enough. In addition, biological reconstruction such as autologous bone graft is recommended for the patients who are younger or suffered from benign tumor. As for the patients who are older, with malignant tumors, underwent adjuvant radiotherapy or chemotherapy, functional reconstruction with bone cement is a good choice for the excellent instantaneous mechanical stability.

\section{MATERIALS AND METHODS}

\section{Data collection}

We retrospectively reviewed our orthopedic database and identified 41 patients who had underwent internal hemipelvectomy of type I or type I + IV for bone tumors at our institution between 2003 and 2015. Of these 11 patients undergoing pelvic resection and reconstruction with insufficient data were excluded from the analysis.

The clinical data, therapy details, and outcomes of the patients were collected. Radiography, CT and MRI studies were used to evaluate the site of the tumor involved and the mechanical outcomes. The study was approved by the ethics committee of the authors' institution.

The study group included 16 male and 14 female with a mean age of 40.7 years (range, 15 to 69 years), a mean body weight of $54.5 \mathrm{~kg}$ (range, 34 to $70 \mathrm{~kg}$ ), a mean height of $166.4 \mathrm{~cm}$ (range, 149 to $177 \mathrm{~cm}$ ), and a mean BMI of 19.6 (range, 12.9 to 23.5) at the time of the index reconstruction. The demographic data and adjuvant therapy details are show in Table 1.

\section{Specific illustration}

The initial tumor location and type of surgical resection was classified as previously reported [4]. A type I lesion involves resection of the ilium, a type IV lesion refers to the lesions involving a portion of sacrum. Combination of type I and type IV depends on the extent of bony invasion by tumor. Implant failure was defined as the breakage or loosening of the implant.

We include the one rod and three screws patients which had one screw fixed in pelvis and two screws in lumbo/sacral vertebra in ORTS group, as for only one screw fixed in supraacetabular. We then include the one rod four screws patients in TRFS group, because of two screws fixed in supraacetabular and lumbo/sacral vertebra respectively.

We define the multiaxial pedicle screws placed through lateral surface of vertebral bodies or pedicle of L4 or L5 as extrapelvic fixation (Figure 2A), and define the screws placed through lateral surface of vertebral bodies or pedicle of sacrum as intrapelvic fixation (Figures 3-4A, 6A). Finally, we divide the ORTS group into two groups: extrapelvic group and intrapelvic group; divide the TRFS group into three groups: extrapelvic+extrapelvic group, extrapelvic+intrapelvic group (Figure 1A, 7A), intrapelvic+intrapelvic group (Figure 8A) depends on the place of the screws. The surgical details, complications and the outcomes of the patients are show in Table 2 .

\section{Surgical procedure}

The resection of the pelvic bone tumor is in accordance with Enneking [40]. Two experienced surgeons performed these surgeries and the procedures. Multiaxial pedicle screws (Click'x, Synthes, Switzerland or M8, Medtronic, USA) were placed through pedicle or lateral surface of vertebral bodies of L4, L5 and sacrum. One or two titanium rods were then used to connect the screws, similar to the methods introduce in the literatures $[10,12]$. The lateral lumbar/sacral vertebral body screw or the vertebral pedicle screw connecting to supraacetabular screw were sometimes applied as indicated (Figures 1-4A, 6-8A).

Part of the patients were only reconstructed with rod-screw system (Figure 2A), while some were also encased in antibiotic-impregnated bone cement (Figures 1A, 4A, 7A). The rest were reconstructed with rod-screw system coupled with autologous bone graft (Figures $1 \mathrm{C}$, $3 \mathrm{~A}, 6 \mathrm{~A}, 8 \mathrm{~A}$ ), such as fibular grafts, iliac crest or bone graft in titanium cages. In some cases, we fixed the bone grafts to the ilium or sacrum by cortical bone screws to increase stability (Figure 1C).

A combination of type I and IV surgical resection was performed in 6 cases. Specific resection ranges of type IV in these 6 cases were introduced as follows, case 4 was received right sagittal hemisacrectomy combined with transverse partial sacrectomy, the right sacral roots 1-5 and left sacral roots 4-5 were sacrificed; case 19 was received right sagittal hemisacrectomy, the right sacral roots 1-3 were sacrificed; case 21 was received left sagittal hemisacrectomy combined with transverse partial sacrectomy, the left sacral roots 1-5 and right sacral roots 3-5 were sacrificed; case 22 was received right sagittal hemisacrectomy, the right sacral roots $1-3$ were sacrificed; case 23 was received right partial sacral ala resection; case 25 was received left partial sacral ala resection.

\section{Statistical analysis}

Kaplan-Meier survival analysis was performed to estimate overall mechanical survival, in which the event was defined as mechanical failure for any reason. Surviving patients or died patients with no implant failure were censored at the last date of follow-up in the analysis of overall mechanical survival. Log rank test was used to compare implant survival rates between different groups. Pooled over strata test was used to compare the overall difference between each groups, and the pairwise for each stratum test was used to compare the difference between 
each two groups. Cox regression was used to analysis the correlation ship between the implant survival rate and the ages. A $p$-value of less than 0.05 was considered to be significant. All statistical analyses were performed using the Statistical Package for the Social Science (SPSS) software, version 22.0 (SPSS Inc., Chicago, IL, USA).

\section{ACKNOWLEDGMENTS}

The authors thank all the research staff for their team collaboration work and all the patients and oncologists involved in this study for their cooperation and support.

\section{CONFLICTS OF INTEREST}

The authors do not have any possible conflicts of interest.

\section{GRANT SUPPORT}

This work was supported by Natural Science Foundation of Zhejiang Province (Y16H160067).

\section{REFERENCES}

1. O'Connor MI, Sim FH. Salvage of the limb in the treatment of malignant pelvic tumors. J Bone Joint Surg Am. 1989; 71:481-94.

2. Wirbel RJ, Schulte M, Mutschler WE. Surgical treatment of pelvic sarcomas: oncologic and functional outcome. Clin Orthop Relat Res. 2001; 390:190-205.

3. Sherman CE, O'Connor MI, Sim FH. Survival, local recurrence, and function after pelvic limb salvage at 23 to 38 years of followup. Clin Orthop Relat Res. 2012; 470: 712-27. DOI: 10.1007/s11999-011-1968-x.

4. Enneking WF, Dunham WK. Resection and reconstruction for primary neoplasms involving the innominate bone. J Bone Joint Surg Am. 1978; 60:731-46.

5. Angelini A, Calabrò T, Pala E, Trovarelli G, Maraldi M, Ruggieri P. Resection and reconstruction of pelvic bone tumors. Orthopedics. 2015; 38:87-93. DOI: 10.3928/0147744720150204-51.

6. Wang B, Xie X, Yin J, Zou C, Wang J, Huang G, Wang Y, Shen J. Reconstruction with modular hemipelvic endoprosthesis after pelvic tumor resection: a report of 50 consecutive cases. PLoS One. 2015; 10:e0127263. DOI: 10.1371/journal.pone.0127263.

7. Ji T, Guo W, Yang RL, Tang XD, Wang YF. Modular hemipelvic endoprosthesis reconstruction-experience in 100 patients with mid-term follow-up results. Eur J Surg Oncol. 2013; 39:53-60. DOI: 10.1016/j.ejso.2012.10.002.

8. Tang X, Guo W, Ji T. Reconstruction with modular hemipelvic prosthesis for the resection of solitary periacetabular metastasis. Arch Orthop Trauma Surg. 2011; 131:1609-15. DOI: 10.1007/s00402-011-1359-5.

9. Beadel GP, McLaughlin CE, Aljassir F, Turcotte RE, Isler MH, Ferguson P, Griffin AM, Bell RS, Wunder JS. Iliosacral resection for primary bone tumors: is pelvic reconstruction necessary. Clin Orthop Relat Res. 2005; 438: 22-9.

10. Nassif NA, Buchowski JM, Osterman K, McDonald DJ. Surgical technique: Iliosacral reconstruction with minimal spinal instrumentation. Clin Orthop Relat Res. 2013; 471: 947-55. DOI: 10.1007/s11999-012-2643-6.

11. Sabourin M, Biau D, Babinet A, Dumaine V, Tomeno B, Anract P. Surgical management of pelvic primary bone tumors involving the sacroiliac joint. Orthop Traumatol Surg Res. 2009; 95:284-92. DOI: 10.1016/j.otsr.2009.04.008.

12. Gebert C, Wessling M, Gosheger G, Aach M, Streitbürger A, Henrichs MP, Dirksen U, Hardes J. Pelvic reconstruction with compound osteosynthesis following hemipelvectomy: A clinical study. Bone Joint J. 2013; 95-B:1410-6. DOI: 10.1302/0301-620X.95B10.31123.

13. Aach M, Gebert C, Ahrens H, Dieckmann R, Gosheger G, Hardes J, Wessling M. The primary stability of pelvic reconstruction after partial supraacetabular pelvic resection due to malignant tumours of the human pelvis: a biomechanical in vitro study. Med Eng Phys. 2013; 35: 1731-5. DOI: 10.1016/j.medengphy.2013.07.014.

14. Krieg AH, Lenze U, Gaston MS, Hefti F. The outcome of pelvic reconstruction with non-vascularised fibular grafts after resection of bone tumours. J Bone Joint Surg Br. 2010; 92:1568-73. DOI: 10.1302/0301-620X.92B11.24893.

15. Nishida J, Shiraishi H, Okada K, Ehara S, Shimamura T. Vascularized iliac bone graft for iliosacral bone defect after tumor excision. Clin Orthop Relat Res. 2006; 447:145-51. DOI: 10.1097/01.blo.0000203485.90711.1b.

16. Hugate R, Sim FH. Pelvic reconstruction techniques. Orthop Clin North Am. 2006; 37:85-97. DOI: 10.1016/j. ocl.2005.08.006.

17. Schwameis E, Dominkus M, Krepler P, Dorotka R, Lang S, Windhager R, Kotz R. Reconstruction of the pelvis after tumor resection in children and adolescents. Clin Orthop Relat Res, 2002; 402:220-35.

18. Kollender Y, Shabat S, Bickels J, Flusser G, Isakov J, Neuman Y, Cohen I, Weyl-Ben-Arush M, Ramo N, Meller I. Internal hemipelvectomy for bone sarcomas in children and young adults: surgical considerations. Eur J Surg Oncol. 2000; 26:398-404. DOI: 10.1053/ejso.1999.0906.

19. Chen $\mathrm{X}, \mathrm{Xu} \mathrm{L}$, Wang Y, Hao Y, Wang L. Image-guided installation of 3D-printed patient-specific implant and its application in pelvic tumor resection and reconstruction surgery. Comput Methods Programs Biomed, 2016; 125:66-78. DOI: 10.1016/j.cmpb.2015.10.020.

20. Bus MP, Boerhout EJ, Bramer JA, Dijkstra PD. Clinical outcome of pedestal cup endoprosthetic reconstruction after 
resection of a peri-acetabular tumour. Bone Joint J. 2014; 96-B:1706-12. DOI: 10.1302/0301-620X.96B12.34622.

21. Enneking WF, Dunham W, Gebhardt MC, Malawar M, Pritchard DJ. A system for the functional evaluation of reconstructive procedures after surgical treatment of tumors of the musculoskeletal system. Clin Orthop Relat Res. 1993; 286:241-6.

22. Ham SJ, Schraffordt Koops H, Veth RP, van Horn JR, Eisma WH, Hoekstra HJ. External and internal hemipelvectomy for sarcomas of the pelvic girdle: consequences of limbsalvage treatment. Eur J Surg Oncol. 1997; 23:540-6.

23. Refaat Y, Gunnoe J, Hornicek FJ, Mankin HJ. Comparison of quality of life after amputation or limb salvage. Clin Orthop Relat Res. 2002; 397:298-305.

24. Mankin HJ, Hornicek FJ. Internal hemipelvectomy for the management of pelvic sarcomas. Surg Oncol Clin N Am. 2005; 14:381-96. DOI: 10.1016/j.soc.2004.11.010.

25. Akiyama T, Clark JC, Miki Y, Choong PF. The nonvascularised fibular graft: a simple and successful method of reconstruction of the pelvic ring after internal hemipelvectomy. J Bone Joint Surg Br. 2010; 92:999-1005. DOI: 10.1302/0301-620X.92B7.23497.

26. Jia YW, Cheng LM, Yu GR, Du CF, Yang ZY, Yu Y, Ding ZQ. A finite element analysis of the pelvic reconstruction using fibular transplantation fixed with four different rod-screw systems after type I resection. Chin Med J (Engl). 2008; 121:321-6.

27. Wang B, Sun P, Xie X, Wu W, Tu J, Ouyang J, Shen J. A novel combined hemipelvic endoprosthesis for periacetabular tumours involving sacroiliac joint: a finite element study. Int Orthop. 2015; 39:2253-9. DOI: 10.1007/ s00264-015-2891-7.

28. Liang H, Li D, Guo W, Yang R, Tang X. Lateral lumbar vertebral body screw predisposes to neuralgia after limb-salvage surgery for pelvic tumors: a single-center, retrospective study of 349 cases. Eur Spine J. 2016; DOI: 10.1007/s00586-016-4685-z.

29. Ozaki T, Hillmann A, Lindner N, Blasius S, Winkelmann W. Chondrosarcoma of the pelvis. Clin Orthop Relat Res. 1997; 337:226-39.
30. Hillmann A, Hoffmann C, Gosheger G, Rödl R, Winkelmann W, Ozaki T. Tumors of the pelvis: complications after reconstruction. Arch Orthop Trauma Surg. 2003; 123:340-4. DOI: 10.1007/s00402-003-0543-7.

31. Ozaki T, Hillmann A, Bettin D, Wuisman P, Winkelmann W. High complication rates with pelvic allografts. Experience of 22 sarcoma resections. Acta Orthop Scand. 1996; 67:333-8.

32. Mankin HJ, Hornicek FJ, Raskin KA. Infection in massive bone allografts. Clin Orthop Relat Res. 2005; 432:210-6.

33. Ogura K, Sakuraba M, Miyamoto S, Fujiwara T, Chuman H, Kawai A. Pelvic ring reconstruction with a double-barreled free vascularized fibula graft after resection of malignant pelvic bone tumor. Arch Orthop Trauma Surg. 2015; 135: 619-25. DOI: 10.1007/s00402-015-2197-7.

34. Nagoya S, Usui M, Wada T, Yamashita T, Ishii S. Reconstruction and limb salvage using a free vascularised fibular graft for periacetabular malignant bone tumours. J Bone Joint Surg Br. 2000; 82:1121-4.

35. Chang DW, Fortin AJ, Oates SD, Lewis VO. Reconstruction of the pelvic ring with vascularized double-strut fibular flap following internal hemipelvectomy. Plast Reconstr Surg. 2008; 121:1993-2000. DOI: 10.1097/PRS.0b013e3181706ff2.

36. Sakuraba M, Kimata Y, Iida H, Beppu Y, Chuman H, Kawai A. Pelvic ring reconstruction with the doublebarreled vascularized fibular free flap. Plast Reconstr Surg. 2005; 116:1340-5.

37. Farhadi J, Valderrabano V, Kunz C, Kern R, Hinterman B, Pierer G. Free fibula donor-site morbidity: clinical and biomechanical analysis. Ann Plast Surg. 2007; 58:405-10. DOI: 10.1097/01.sap.0000241948.36784.4e.

38. Tuli SM. Bridging of bone defects by massive bone grafts in tumorous conditions and in osteomyelitis. Clin Orthop Relat Res. 1972; 87:60-73.

39. Krieg AH, Hefti F. Reconstruction with nonvascularised fibular grafts after resection of bone tumours. J Bone Joint Surg Br. 2007; 89:215-21. DOI: 10.1302/0301-620X.89B2.17686.

40. Enneking WF. A system of staging musculoskeletal neoplasms. Clin Orthop Relat Res. 1986; 204:9-24. 